\title{
Comment les enseignants immigrants formés à l'étranger vivent-ils les relations interpersonnelles et professionnelles à leur entrée dans le milieu scolaire québécois?
}

\author{
Aline Niyubahwe, Joséphine Mukamurera et Geneviève Sirois
}

Volume 8, numéro 2, 2018

L'école à l'épreuve de la diversité ethnoculturelle de son personnel : regards compréhensifs croisés

URI : https://id.erudit.org/iderudit/1066950ar

DOI : https://doi.org/10.7202/1066950ar

Aller au sommaire du numéro

Éditeur(s)

Alterstice

ISSN

1923-919X (numérique)

Découvrir la revue

Citer cet article

Niyubahwe, A., Mukamurera, J. \& Sirois, G. (2018). Comment les enseignants immigrants formés à l'étranger vivent-ils les relations interpersonnelles et professionnelles à leur entrée dans le milieu scolaire québécois ? Alterstice, 8(2), 25-36. https://doi.org/10.7202/1066950ar

\section{Résumé de l'article}

Le nombre d'enseignants immigrants formés à l'étranger (EIFÉ) ne cesse de croître dans les milieux scolaires québécois. Malgré leur expérience d'enseignement acquise dans leurs pays d'origine, ils font figure de débutants dans le nouveau système scolaire dont ils ne connaissent pas les politiques, le fonctionnement, ni la culture professionnelle. Ainsi, leur socialisation peut comporter d'énormes défis puisqu'ils doivent à la fois s'intégrer dans leur milieu de travail dont les repères leur échappent, tout en étant appelés à coopérer et à travailler de concert avec les autres acteurs de la scène scolaire. Cet article a pour objectif de décrire et comprendre comment ces enseignants vivent les relations interpersonnelles et professionnelles lors de leurs premières années d'embauche dans le milieu scolaire québécois. Le cadre d'analyse de notre recherche s'appuie sur le concept d'insertion professionnelle, particulièrement sur le plan de la socialisation organisationnelle. Les données analysées sont issues d'une étude qualitative d'inspiration phénoménologique menée au moyen d'entrevues semi-dirigées auprès de 13 EIFÉ des régions de Montréal et de Sherbrooke. Les résultats suggèrent que les EIFÉ vivent souvent des problématiques frustrantes, marquées par un accueil froid, l'indifférence, l'isolement, la méfiance, l'absence de soutien et de collaboration ainsi que la non-reconnaissance professionnelle. Quelques-uns rapportent des expériences positives, ce qui laisse comprendre que la situation peut varier d'une école à l'autre en fonction des caractéristiques contextuelles et des qualités personnelles des EIFÉ. Ces résultats sont discutés et des pistes d'action sont proposées pour le milieu scolaire.
Droits d'auteur (c) Aline Niyubahwe, Joséphine Mukamurera et Geneviève Sirois, 2018
Ce document est protégé par la loi sur le droit d'auteur. L’utilisation des services d'Érudit (y compris la reproduction) est assujettie à sa politique d'utilisation que vous pouvez consulter en ligne.

https://apropos.erudit.org/fr/usagers/politique-dutilisation/ 


\title{
7
}

ARTICLE THÉMATIQUE

\section{Comment les enseignants immigrants formés à l'étranger vivent-ils les relations interpersonnelles et professionnelles à leur entrée dans le milieu scolaire québécois?}

Aline Niyubahwe $^{1}$, Joséphine Mukamurera ${ }^{2}$ et Geneviève Sirois ${ }^{1}$

\begin{abstract}
Résumé
Le nombre d'enseignants immigrants formés à l'étranger (EIFÉ) ne cesse de croître dans les milieux scolaires québécois. Malgré leur expérience d'enseignement acquise dans leurs pays d'origine, ils font figure de débutants dans le nouveau système scolaire dont ils ne connaissent pas les politiques, le fonctionnement, ni la culture professionnelle. Ainsi, leur socialisation peut comporter d'énormes défis puisqu'ils doivent à la fois s'intégrer dans leur milieu de travail dont les repères leur échappent, tout en étant appelés à coopérer et à travailler de concert avec les autres acteurs de la scène scolaire. Cet article a pour objectif de décrire et comprendre comment ces enseignants vivent les relations interpersonnelles et professionnelles lors de leurs premières années d'embauche dans le milieu scolaire québécois. Le cadre d'analyse de notre recherche s'appuie sur le concept d'insertion professionnelle, particulièrement sur le plan de la socialisation organisationnelle. Les données analysées sont issues d'une étude qualitative d'inspiration phénoménologique menée au moyen d'entrevues semi-dirigées auprès de 13 EIFÉ des régions de Montréal et de Sherbrooke. Les résultats suggèrent que les EIFÉ vivent souvent des problématiques frustrantes, marquées par un accueil froid, l'indifférence, l'isolement, la méfiance, l'absence de soutien et de collaboration ainsi que la non-reconnaissance professionnelle. Quelques-uns rapportent des expériences positives, ce qui laisse comprendre que la situation peut varier d'une école à l'autre en fonction des caractéristiques contextuelles et des qualités personnelles des EIFÉ. Ces résultats sont discutés et des pistes d'action sont proposées pour le milieu scolaire.
\end{abstract}

\section{Rattachement des auteures}

${ }^{1}$ Université du Québec en Abitibi-Témiscamingue, Canada; ${ }^{2}$ Université de Sherbrooke, Sherbrooke, Canada

\section{Correspondance}

aline.niyubahwe@uqat.ca

\section{Mots clés}

enseignant immigrant formé à l'étranger, relations professionnelles, diversité ethnoculturelle, intégration, socialisation organisationnelle

\section{Pour citer cet article}

Niyubahwe, A., Mukamurera, J. et Sirois, G. (2018). Comment les enseignants immigrants formés à l'étranger vivent-ils les relations interpersonnelles et professionnelles à leur entrée dans le milieu scolaire québécois? Alterstice, 8(2), 25-36. 


\section{Introduction}

Parmi le nouveau personnel enseignant des écoles québécoises, on trouve de plus en plus d'enseignants immigrants formés à l'étranger (EIFÉ). La plupart intègrent des établissements scolaires ayant des normes, des valeurs et un mode de fonctionnement autres que ce à quoi ils étaient habitués dans leur pays d'origine. Ils ont donc besoin d'être socialisés en raison de l'écart entre la culture organisationnelle de leurs établissements d'origine et celle de leur nouvel environnement de travail. Selon Lacroix (2014), la réussite du processus de socialisation des immigrants dépend entre autres de la qualité des relations avec les membres de leur nouvel environnement de travail. Ainsi, s'intéresser aux relations interpersonnelles et professionnelles que vivent les EIFÉ lors de leur entrée dans le milieu scolaire québécois devient un enjeu important pour leur intégration socioprofessionnelle et leur rétention. En effet, Collin et Camaraire (2013) soutiennent qu'une bonne intégration des EIFÉ " peut influencer d'une part la qualité de leur enseignement et, d'autre part, la qualité du modèle de diversité qu'ils symbolisent aux yeux des élèves » (p. 50). Au Québec, le ministère de l'Éducation, du Loisir et du Sport souligne également l'importance "d'exposer les élèves issus de l'immigration à des modèles d'intégration réussie auxquels ils peuvent s'identifier et à l'aide desquels ils peuvent se projeter dans l'avenir en tant que citoyens actifs de la société québécoise " (Gouvernement du Québec, 2014, p. 5). II est donc important de se pencher sur l'intégration des enseignants immigrants au milieu scolaire. C'est l'objet de cet article.

\section{Problématique de la recherche}

L'embauche d'enseignants immigrants n'est pas un phénomène récent au Québec. Cependant, en raison de la pénurie actuelle d'enseignants, le nombre d'EIFÉ ne cesse de croître dans les milieux scolaires québécois. Plus de 350 autorisations d'enseigner sont décernées chaque année aux EIFÉ (Morrissette, Charara et Boily, 2015). Lorsqu'ils sont embauchés, ceux-ci intègrent à la fois un nouveau système d'enseignement et un nouvel environnement de travail. Malgré l'expérience d'enseignement acquise dans leurs pays d'origine, ils font figure de débutants dans le nouveau système scolaire, dont ils ne connaissent ni les politiques, ni le fonctionnement, ni la culture professionnelle (Niyubahwe, Mukamurera et Jutras, 2018). Ils doivent alors s'imprégner de la culture de l'école, se familiariser avec leur nouvel environnement de travail, tout en étant appelés à coopérer et à travailler de concert avec l'équipe-école, les parents et les membres de l'équipe pédagogique (Gouvernement du Québec, 2001). Leur socialisation peut comporter des défis plus ou moins importants en fonction de l'écart entre leurs attentes et celles de ce nouvel environnement de travail.

Une recension exhaustive des écrits (Niyubahwe, Mukamurera et Jutras, 2013) montre que les EIFÉ font face à des difficultés d'insertion professionnelle qui leur sont propres, notamment des difficultés d'accès à l'emploi, des difficultés relatives au manque de reconnaissance des compétences acquises dans leurs pays d'origine, des difficultés d'intégration dans le milieu de travail ainsi que des difficultés relatives à l'appropriation du rôle professionnel spéficique à leur nouveau contexte socioprofessionnel. Elle permet aussi de faire ressortir certains éléments favorables à leur insertion. Dans le cadre de cet article, l'attention est mise d'une part sur les difficultés et d'autre part sur les facteurs favorables à leur intégration au milieu scolaire.

\section{Les difficultés d'intégration au milieu scolaire}

Plusieurs recherches faites au Canada (Bascia, 1996 ; Duchesne, 2017 ; Jabouin, 2018 ; Morrissette et Demazière, 2018; Phillion, 2003), en Israël (Remennick, 2002) et en Australie (Peeler et Jane, 2005; Reid, 2005) montrent que les EIFÉ font face à plusieurs défis d'intégration à leur milieu de travail.

Au Canada, les EIFÉ ayant participé à une étude faite en Ontario (Duchesne, 2017) affirment avoir reçu un accueil froid, voire " hostile " lors de leur entrée dans les écoles en milieu rural. De plus, toutes les études faites en Ontario (Bascia, 1996; Duchesne, 2017 ; Jabouin, 2018 ; Phillion, 2003 ; Wang 2003) aboutissent à la conclusion selon laquelle certains EIFÉ, particulièrement ceux appartenant aux minorités visibles, vivent de l'isolement social, voire du racisme et de la discrimination à l'intérieur de l'école. Par exemple, les participants à l'étude de Jabouin (2018) déplorent non seulement que leurs collègues blancs refusent de s'asseoir avec eux et de leur parler lorsqu'ils se trouvent ensemble dans la salle des enseignants, mais aussi qu'ils tiennent des propos déplaisants à 
leur égard, ce qui rend leurs relations très tendues. Aussi, un participant à cette étude rapporte que ses collègues tiennent difficilement compte de son opinion lors d'un travail collaboratif, ce qu'il attribue à des préjugés négatifs à son égard. Le problème de préjugés s'observe aussi dans les relations avec les parents. À cet égard, une participante d'origine africaine se plaint que certains parents d'élèves s'imaginaient difficilement qu'elle a les compétences requises pour enseigner les sciences. Quant aux participants à l'étude de Wang (2003), ils déplorent la distance, l'indifférence et la méfiance du personnel de direction à leur égard. D'ailleurs, ces participants affirment que leur période d'intégration a été marquée par beaucoup de frustrations, d'anxiété, de stress et de pression. La pression de certains parents et directions à l'égard des EIFÉ est aussi observée dans l'étude faite au Québec par Morrissette et Demazière (2018).

En Israël, l'étude de Remennick (2002) révèle que les enseignants israéliens considèrent leurs collègues d'origine russe comme une menace à leur emploi, ce qui génère des relations conflictuelles et l'isolement de ces enseignants d'origine russe. Selon les participants à cette étude, les enseignants israéliens refusent de collaborer avec eux et de leur servir de mentors. Ils déplorent aussi l'absence de collaboration de la part des conseillers scolaires et des parents d'élèves, ces derniers prenant toujours le parti de leurs enfants en cas de litige. En Australie, l'étude de Peeler et Jane (2005) sur l'importance du mentorat dans la transition professionnelle du personnel enseignant immigrant montre quant à elle qu'un échec dans la relation entre un enseignant immigrant et son mentor peut affecter du même coup ses relations avec les autres membres du personnel enseignant.

\section{Les facteurs favorables à l'intégration dans le milieu scolaire}

Parallèlement, différentes études montrent que plusieurs facteurs facilitent l'intégration socioprofessionnelle des EIFÉ, en particulier un accueil bienveillant, une ouverture des collègues et du personnel de direction qui se matérialise par l'établissement des relations harmonieuses, collaboratives et d'entraide, un accompagnement par un mentor (Deters, 2006; Jabouin, 2018 ; Morrissette et Demazière, 2018; Peeler et Jane, 2005), une acceptation par la communauté scolaire et par les parents ainsi que le fait de travailler dans un milieu culturellement diversifié (Deters, 2006). En outre, les études de Deters (2006) et Jabouin (2018) montrent que certaines caractéristiques personnelles des EIFÉ comme l'ouverture aux autres et à la culture du milieu scolaire, la capacité d'autocritique, le fait de ne pas tenir compte des on-dit et l'implication dans leur milieu scolaire contribuent au succès de leur intégration et au développement d'un sentiment d'appartenance à leur communauté scolaire. Par exemple, les participants à l'étude de Jabouin (2018) soulignent que leur ouverture aux enseignants natifs leur a permis non seulement de tisser de bonnes relations, mais aussi de bénéficier du soutien et d'un accompagnement nécessaire à l'apprentissage de leur rôle professionnel dans le nouveau contexte.

En fin de compte, il ressort de ce qui précède que l'intégration des EIFÉ à leur nouvel environnement professionnel s'accompagne de défis, voire de difficultés, et que certains facteurs organisationnels, relationnels et personnels peuvent jouer un rôle important dans la qualité de leur expérience d'intégration. Eu égard à cette problématique, cet article a pour objectif de décrire et comprendre comment les ElFÉ vivent les relations interpersonnelles et professionnelles lors de leurs premières années d'embauche dans le milieu scolaire québécois.

\section{Cadre de référence : la socialisation organisationnelle}

De la même manière que les enseignants débutants, les EIFÉ vivent un processus d'insertion professionnelle lorsqu'ils entrent dans le milieu scolaire québécois. Selon Mukamurera (2018), l'insertion professionnelle comporte cinq dimensions interdépendantes que sont l'intégration en emploi, l'affectation spécifique et les conditions de la tâche, la socialisation organisationnelle, la professionnalité et la dimension personnelle et psychologique. Dans le cadre de cet article, c'est la dimension de la socialisation organisationnelle de l'insertion qui est mobilisée pour la constitution et l'analyse des données visant à éclairer notre objet d'étude.

Selon Mukamurera (2018), la socialisation organisationnelle fait référence à l'intégration dans le milieu du travail, l'adaptation à la culture de l'école et l'intégration à l'équipe-école. Bengle (1993) définit la socialisation organisationnelle comme un « processus par lequel une personne en arrive à apprécier les valeurs, les habiletés, les comportements attendus et les connaissances sociales essentielles afin d'assumer un rôle dans l'organisation et d'y participer en tant que membre» (p. 15). Plusieurs auteurs (Duchesne, 2002 ; Lacroix, 2014 ; Lapointe, 2013) 
considèrent que l'accueil constitue la première étape de la socialisation, car c'est par lui qu'on entre en interaction avec les nouveaux employés. Or, selon Lacroix (2014, p. 35), "l'accueil ne pourrait avoir lieu s'il manque d'ouverture à l'égard d'un nouvel arrivant ". Il précise en outre que l'intégration " se vit dans les relations entre collègues de travail et entre supérieurs et employés » (p. 26). Dans le cas des enseignants, elle se vit aussi dans les relations avec les autres acteurs de la scène scolaire, dont les professionnels non enseignants ainsi que les parents. Ainsi, les interactions positives " favoriseraient l'acquisition d'un comportement de rôle approprié, le développement de compétences au travail, l'ajustement aux normes et valeurs du groupe de travail, la compréhension des normes et des procédures organisationnelles " (Reichers, 1987, cité dans Fabre et Roussel, 2013, p. 6). Garant, Lavoie, Hensler et Beauchesne (1999, cités dans Portelance, Martineau et Presseau, 2008, p. 128) soutiennent que la réussite du processus de socialisation organisationnelle nécessite "la création d’un environnement propice aux échanges, des relations de confiance et de respect mutuel, la prise en compte du point de vue de son partenaire ainsi que la valorisation de ses propres connaissances ».

La socialisation des EIFÉ peut constituer un défi de taille. D’une part, leur cadre de référence peut être différent, voire très éloigné de celui des autres membres de la communauté scolaire, ce qui peut générer des incompréhensions mutuelles et entraver la qualité des relations. D’autre part, il peut être difficile de tenir compte ou de valoriser les connaissances de quelqu'un qui ne partage pas le même cadre de référence. Ainsi, les enseignants vont juger leurs collègues immigrants à partir de leur cadre de référence, en tenant compte de ce qui est admis et valorisé dans leur milieu scolaire. Or, ce qui est admis et valorisé ici ne l'est pas nécessairement ailleurs, notamment dans les systèmes éducatifs des pays d'origine des ElFÉ. À cet égard, Lacroix (2014) note que l'arrivée d'un immigrant dans son nouveau milieu de travail peut être perçue comme une « menace "à la culture et à l’identité. Campoy, Waxin, Davoine, Charles-Pauvers, Commeiras et Goudarzi,. (2005, p. 358) soulignent aussi que l'intégration dans une nouvelle culture organisationnelle comporte plusieurs défis, particulièrement « si la culture organisationnelle de l'organisation hôte est éloignée de celle de l'organisation d'origine ". Tout comme l'indique Lacroix (2014) à propos des immigrants, l'intégration des ElFÉ implique non seulement leur ouverture aux membres de la communauté scolaire d'accueil et à leur culture, mais aussi l'acceptation et la reconnaissance sociale et professionnelle de ces derniers à leur égard. Au Québec, l'intégration organisationnelle des EIFÉ paraît cruciale également par le fait que la concertation et la collaboration entre les acteurs de la scène scolaire sont mises de l'avant pour mieux soutenir l'apprentissage des élèves et l'atteinte des objectifs de l'école (Gouvernement du Québec, 2001 et 2006). Il serait alors difficile pour les EIFÉ de réussir leur intégration et leur rôle professionnel sans entretenir des relations interpersonnelles et professionnelles harmonieuses avec les autres membres de l'équipeécole et les parents de leurs élèves. Lapointe (2013, p. 1) soutient d'ailleurs que les institutions tireraient profit d'une attention particulière portée au processus de socialisation s'ils veulent retenir leurs employés.

\section{Cadre méthodologique}

L'objectif étant de décrire et comprendre comment les EIFÉ vivent les relations interpersonnelles et professionnelles durant les premières années d'embauche dans le milieu scolaire québécois, l'approche qualitative d’inspiration phénoménologique a été privilégiée, car elle permet de décrire les expériences telles qu'elles sont vécues par les participants (Giorgi, 1997). Nous avons recruté 13 participants dans les régions de Montréal et de Sherbrooke en fonction de leur pertinence théorique. L'échantillonnage était donc intentionnel (Karsenti et SavoieZajc, 2004) et trois critères de base ont été retenus. Ils devaient être avant tout des ElFÉ avec sept ans ou moins de résidence au Québec. De plus, ils devaient avoir eu une formation initiale à l'enseignement, une qualification et une expérience d'enseignement dans leur pays d'origine. Enfin, ils devaient avoir au moins une année d'expérience d'enseignement au Québec, afin d'avoir accès à des expériences significatives et variées pour éclairer le phénomène à l'étude.

L'échantillon est constitué de 9 femmes et de 4 hommes. Le niveau de formation de ces enseignants varie de la licence (baccalauréat au Québec) au doctorat en passant par la maîtrise. Leur expérience d’enseignement dans leur pays d'origine varie de 3 à 20 ans, tandis que l'expérience au Québec varie d'1 à 6 ans. Le tableau 1 présente les caractéristiques détaillées des participants. Des pseudonymes leur ont été attribués pour préserver leur anonymat.

Alterstice - Revue Internationale de la Recherche Interculturelle, vol. $8, n^{\circ} 2$ 
Tableau 1 : Caractéristiques des participants

\begin{tabular}{|c|c|c|c|c|c|c|}
\hline Participant & Origine & $\begin{array}{l}\text { Champ et niveau de } \\
\text { formation }\end{array}$ & $\begin{array}{l}\text { Années } \\
\text { de } \\
\text { résidence }\end{array}$ & $\begin{array}{c}\text { Niveau et lieu } \\
\text { d'enseignement }\end{array}$ & $\begin{array}{c}\text { Expérience } \\
\text { Pays } \\
\text { d'origine }\end{array}$ & $\begin{array}{c}\text { nseignement } \\
\text { Québec }\end{array}$ \\
\hline Dora & Afrique subsaharienne & $\begin{array}{l}\text { Sciences et technologies, } \\
\text { Maîtrise }\end{array}$ & 3 ans & $\begin{array}{l}\text { Secondaire, } \\
\text { Montréal }\end{array}$ & 15 ans & 2 ans \\
\hline Prima & Afrique subsaharienne & $\begin{array}{l}\text { Français langue seconde, } \\
\text { Maîtrise }\end{array}$ & 2 ans & $\begin{array}{l}\text { Secondaire } \\
\text { Sherbrooke }\end{array}$ & 13 ans & 1 an \\
\hline Olivia & Afrique subsaharienne & $\begin{array}{l}\text { Littérature africaine, } \\
\text { Licence }\end{array}$ & 6 ans & $\begin{array}{l}\text { Secondaire, } \\
\text { Montréal }\end{array}$ & 5 ans & 2 ans \\
\hline Nadia & Maghreb & $\begin{array}{l}\text { Français langue seconde, } \\
\text { Maîtrise }\end{array}$ & 4 ans & $\begin{array}{l}\text { Secondaire, } \\
\text { Montréal }\end{array}$ & 20 ans & 3 ans \\
\hline Latifa & Maghreb & $\begin{array}{l}\text { Didactique du français et } \\
\text { des maths, Maîtrise }\end{array}$ & 6 ans & $\begin{array}{l}\text { Secondaire, } \\
\text { Montréal }\end{array}$ & 6 ans & 3,5 ans \\
\hline Francis & Afrique subsaharienne & $\begin{array}{l}\text { Physique, } \\
\text { Maîtrise }\end{array}$ & 2 ans & $\begin{array}{l}\text { Secondaire, } \\
\text { Sherbrooke }\end{array}$ & 12 ans & 1 an \\
\hline Karim & Maghreb & Linguistique, Doctorat & 4 ans & $\begin{array}{l}\text { Secondaire, } \\
\text { Montréal }\end{array}$ & 17 ans & 3 ans \\
\hline André & Afrique subsaharienne & $\begin{array}{l}\text { Didactique des maths, } \\
\text { Maîtrise }\end{array}$ & 7 ans & $\begin{array}{l}\text { Secondaire, } \\
\text { Montréal }\end{array}$ & 14 ans & 6 ans \\
\hline Mariya & Europe de l'Est & $\begin{array}{l}\text { Linguistique, } \\
\text { Maîtrise }\end{array}$ & 7 ans & $\begin{array}{l}\text { Primaire } \\
\text { (classe d'accueil), } \\
\text { Montréal }\end{array}$ & 3 ans & 5 ans \\
\hline Eva & Europe de l'Est & $\begin{array}{l}\text { Français langue seconde, } \\
\text { Licence }\end{array}$ & 5 ans & $\begin{array}{l}\text { Primaire } \\
\text { (classe d'accueil), } \\
\text { Montréal } \\
\end{array}$ & 20 ans & 4 ans \\
\hline Michael & Caraïbes & $\begin{array}{l}\text { Enseignement de } \\
\text { l'histoire, } \\
\text { Licence }\end{array}$ & 5 ans & $\begin{array}{l}\text { Secondaire, } \\
\text { Montréal }\end{array}$ & 4 ans & 3 ans \\
\hline Rocio & Amérique Latine & $\begin{array}{l}\text { Enseignement de } \\
\text { l'espagnol, } \\
\text { Licence }\end{array}$ & 7 ans & $\begin{array}{l}\text { Primaire, } \\
\text { Sherbrooke }\end{array}$ & 17 ans & 5 ans \\
\hline Alessandra & Amérique Latine & $\begin{array}{l}\text { Adaptation scolaire et } \\
\text { sociale, } \\
\text { Licence }\end{array}$ & 7 ans & $\begin{array}{l}\text { Secondaire, } \\
\text { Sherbrooke }\end{array}$ & 16 ans & 5 ans \\
\hline
\end{tabular}

La collecte de données a été faite au moyen d'entrevues semi-dirigées entre août 2011 et mai 2012, dans le cadre d'une recherche doctorale. Les thèmes suivants ont été abordés: la formation antérieure et l'expérience d'enseignement, le parcours migratoire, l'adaptation au pays d'accueil, l'insertion en emploi, l'intégration dans le milieu scolaire et l'intégration au rôle professionnel. Les entrevues, d'une durée de 60 à 90 minutes, ont été transcrites intégralement et analysées en suivant les orientations de l'analyse phénoménologique (Bachelor et Joshi, 1986; Giorgi, 1997). Plus concrètement, chacun des verbatim a été lu et relu afin de nous rapprocher du phénomène à l'étude et d'en saisir le sens (Bachelor et Joshi, 1986). Nous avons alors procédé à l'identification et à la délimitation des unités de signification qui émergeaient du texte. Par la suite, il a été question de " délimiter les énoncés redondants et d'articuler le thème principal exprimé autour d'une ou de plusieurs unités naturelles » (Bachelor et Joshi, 1986, p. 51). Comme le suggèrent ces auteurs, nous avons formulé les thèmes centraux dans le langage utilisé par les participants. Après la délimitation des thèmes centraux, nous avons rédigé le récit d'expérience spécifique à chaque participant. Ce dernier a été par la suite invité à le valider et, s'il y avait lieu, à fournir des corrections. Enfin, nous avons analysé les thèmes centraux sur l'ensemble des récits pour repérer des convergences, des différences ou des particularités.

Dans cet article, nous présentons essentiellement les résultats relatifs à l'expérience d'intégration dans le milieu de travail. L'examen du corpus en rapport avec cette dimension fait ressortir deux principaux thèmes: vécu de l'accueil et vécu des relations avec les membres de la communauté scolaire. 


\section{Résultats : vécu de l'accueil}

Trois enseignantes (Dora, Mariya et Rocio) estiment avoir reçu un accueil bienveillant de la direction lors de leur entrée dans le milieu scolaire. Par exemple, après l'accueil, les directrices de ces trois enseignantes ont pris le temps de les présenter à leurs collègues, comme l'exprime Dora : « j'ai été bien accueillie par la directrice, qui m'a présentée à tous les autres profs le lendemain, lors d’une assemblée de la rentrée scolaire. "Quant à Rocio, sa directrice l'a non seulement présentée aux élèves, mais elle lui a aussi permis de faire appel à elle en cas de problème tout en la rassurant qu'elle repasserait voir si tout allait bien.

Contrairement à ces trois enseignantes, les autres affirment avoir vécu un accueil froid, voire absent, comme en témoigne Michaël : "Quand tu arrives à l'école le premier jour, il n’y a rien qui se passe. On te dit : "Voilà ton horaire" ". Ces enseignants étaient déçus et choqués de voir qu'ils ne sont pas accueillis comme dans leurs pays d'origine : " j'ai été choquée par la façon dont on m'a accueillie. Je m'attendais à ce que la directrice ou son adjoint me fasse visiter l'école, me présente à la classe et aux collègues comme dans mon pays » (Prima). Ainsi, ils vivent le sentiment d'être laissés à eux-mêmes puisqu'ils doivent se débrouiller pour trouver le local de classe et s'introduire seuls en classe, comme l'exprime Karim : "J'ai donc rencontré les élèves seul pour la première fois et ce fut un grand défi pour moi. " Cette absence d'accueil nuit à l'intégration socioprofessionnelle en raison du manque d'information sur l'organisation, le fonctionnement et les ressources pertinentes de l'école. L'intégration à l'équipe-école est ainsi délicate, comme le témoigne Prima : « Vous arrivez là comme un cheveu sur la soupe, les gens vous regardent, mais ne savent pas qui vous êtes ".

\section{Résultats : vécu des relations avec les membres de la communauté scolaire}

\section{Rapports avec la direction}

Certains participants affirment avoir vécu des relations harmonieuses avec leurs directions d'école, ce qui a contribué à la réussite de leur intégration socioprofessionnelle. Par exemple, Dora se réjouit de l'attitude positive de sa directrice qui, après l'avoir chaleureusement accueillie et présentée aux autres professeurs, a facilité l'établissement des liens avec les professeurs intervenant au même cycle et dans la même discipline : " Cette directrice a beaucoup facilité le contact en me donnant les noms des autres profs... surtout celle qui enseignait le secondaire 2 comme moi. Vraiment, on s'est lié d'amitié ce même jour » (Dora). Sur le plan professionnel, les relations harmonieuses se traduisent par le soutien, la rétroaction positive, la valorisation de leur travail et les encouragements de leur direction d'école. Par exemple, vu l'ampleur des difficultés auxquelles elle a fait face la première année, Mariya estime qu'elle n'aurait pas survécu, n'eût été le soutien et les encouragements de sa directrice : " C'est grâce à ma directrice que je n'ai pas décroché la première année. Elle apprécie beaucoup mon travail » (Mariya). Quant à Nadia et Dora, elles ont été marquées par l'attitude attentive de leur direction et les rétroactions constructives reçues. Par exemple, après avoir reçu des remarques de la part de la direction sur son problème de gestion de classe, Nadia a apprécié que sa direction ait aussi, par la suite, reconnu l'amélioration réalisée : « Le directeur m'a convoquée dans son bureau pour me dire que je fais un bon travail. Ça m'a beaucoup encouragée »(Nadia). Rocio et Alessandra soulignent aussi que leurs directions reconnaissent et valorisent la qualité du travail des enseignants par des rétroactions positives. Toutes ces enseignantes témoignent que leurs directions étaient bienveillantes et favorables à leur réembauche à l'école.

Par contre, d'autres EIFÉ estiment que les relations moins harmonieuses avec la direction ou la direction adjointe ont nui à leur intégration socioprofessionnelle. Le manque d'ouverture fait que certaines directions sont moins tolérantes aux différences. À cet égard, Olivia et Éva affirment avoir reçu des remarques négatives de la part de leur direction à cause de leur accent. Par exemple, lors de leur première rencontre, la directrice d'Olivia lui a dit ouvertement que son accent n'était pas convenable pour un professeur de français : " elle m'a appelée et m'a dit : "Toi, tu enseignes le français, mais ce ton-là, ça ne marche pas" ". Olivia s'est alors sentie diminuée et rejetée. Cette attitude de la direction ne contribue pas à créer un climat propice aux échanges et inhibe l'intérêt à demander de l'aide en cas de difficulté : " c'était difficile de me confier à elle ou de lui demander de l'aide alors qu'elle m'avait rejetée au début " (Olivia). Cette enseignante affirme d’ailleurs que cette directrice lui a refusé des heures de suppléances supplémentaires alors qu'elle y avait droit, ce qui l'a amené à penser qu'elle les lui refusait à cause de son accent. Les participants déplorent aussi l'attitude distante de certaines directions qui les laissent à 
eux-mêmes sans aucun soutien ni conseil lors de conflits avec les parents d'élèves: "Je n'ai pas aimé ce comportement un peu offensant de la directrice, car elle m'a mis en face des parents, puis elle est restée en retrait » (Michaël). Malgré le renouvellement de son contrat, Michaël a préféré chercher du travail ailleurs pour sortir de ce climat plutôt négatif. Dans certains cas, les relations conflictuelles avec la direction ou la direction adjointe peuvent être sources d'une mauvaise évaluation de l'ElFÉ. Latifa et Éva déplorent avoir été évaluées négativement par leurs directions adjointes, qui pourtant n'avaient jamais mis les pieds dans leur classe. Latifa pense d'ailleurs que cette évaluation injuste serait due à un petit incident où elle aurait blessé sa directrice adjointe sans s'en rendre compte : "Un jour, la directrice adjointe a dit : "Ferme les lumières !" Comme j'ai l'habitude de corriger les élèves, j'ai dit automatiquement sans réfléchir "Éteins les lumières" ". Probablement que cela l'a beaucoup choquée " (Latifa). Elle raconte d'ailleurs que suite à cet incident, la situation allait de mal en pis tout au long de l'année : "C'était vraiment monstrueux. [...] chaque fois que je lui disais bonjour, elle me regardait puis elle tournait la tête ».

\section{Rapports avec les collègues}

Tous les participants soulignent que leur expérience varie d'une école à l'autre en fonction de son climat, de l'ouverture de ses membres à la diversité ethnoculturelle et de leurs qualités personnelles.

Ainsi, une école où la direction est accueillante favorise l'établissement des liens et une culture de collaboration entre collègues. C'est ce qu'ont vécu cinq enseignants (Dora, Rocio, Mariya, Nadia, André). Ces enseignants affirment que leurs directions ou directions adjointes ont facilité le réseautage avec les collègues et qu'ils ont par la suite bénéficié d'une bonne collaboration de ces derniers. Par exemple, les collègues de Mariya lui ont permis d'aller observer dans leurs classes pour voir comment ça se passe. De plus, ils lui ont donné du matériel pédagogique pour faciliter ses premières leçons. Elle se réjouit d'ailleurs de leur esprit d'équipe : «On travaille normalement en équipe et il y a plus de collaboration ». Dora et Nadia apprécient les rétroactions positives de certains de leurs collègues qui reconnaissaient leur progrès notamment dans la gestion de classe : "Ce sont eux qui venaient me dire que je fais un travail formidable. Cela m'a beaucoup encouragée, car moralement j'étais épuisée " (Nadia). Les relations conviviales et de collégialité entre collègues se concrétisent aussi par l'entraide et l'acceptation : " on échange des évaluations et on se prête des documents " (André); "On dinait ensemble et des fois on s'échangeait des affaires »(Dora). Dans le cas de Rocio, enseignante d'espagnol, ses collègues se soucient de son intégration linguistique en l'aidant à peaufiner son français : « Ils me donnent de bonnes rétroactions et me corrigent quand il le faut ". Les relations positives avec les collègues facilitent le développement d'un sentiment d'appartenance à l'école et le désir de pouvoir continuer à y travailler. C'est ce qu'atteste Nadia : " J'aimerais rester dans cette école, car je trouve que l'ambiance est bonne ".

Au contraire, quatre enseignants (Karim, Alessandra, Prima et Latifa) travaillant dans les écoles où la direction ne favorise pas l'instauration d'un climat favorable aux échanges et à la collaboration ont vécu des expériences négatives avec leurs collègues. Karim et Alessandra affirment que certains collègues transmettaient de l'information à la direction au sujet de leur travail. Alessandra avait même le sentiment d'être surveillée : "lls étaient comme des inspecteurs, ils surveillaient comment je faisais mon travail ». Tout en reconnaissant le soutien de certains collègues, Karim regrette l'attitude d'autres qui, au lieu de lui partager leurs stratégies en gestion de classe, transmettaient l'information à la direction: "Au bout d'un mois, la directrice est venue me dire : “Il faut faire attention parce qu'il y a des enseignants qui se plaignent de votre classe. Là, à un moment donné, je peux vous remercier" ». Karim n’a pas apprécié cette attitude de la direction, car, selon lui, elle aurait pu venir observer les conditions dans lesquelles il travaillait avant de le menacer sur la base des « on-dit ».

Quant à Latifa et Prima, elles disent avoir subi du racisme et de la discrimination de la part de collègues. Par exemple, lorsque Latifa, enseignante musulmane voilée, s'est présentée pour la première fois dans la salle des professeurs pour dîner avec une collègue québécoise qui l'avait invitée, une autre enseignante qui était assise à côté de sa collègue a tout de suite arrêté de parler puis elle est partie sans rien dire. Latifa affirme s'être sentie rejetée, mais elle a continué de jaser comme si rien n'était pour ne pas indisposer sa collègue. Quelques minutes plus tard, d'autres collègues sont venus s'asseoir sur la même table et, au bout d'un certain temps, ils ont commencé à critiquer les parents qui obligent leurs enfants à faire le jeûne du ramadan, puis ils ont poursuivi sur la 
religion musulmane et la polygamie des hommes musulmans, jusqu'à tenter de la provoquer en entrant dans sa vie privée : "Une m'a prise par la main et m'a dit: "Et toi, ton mari a combien de femmes ?". " Ce jour-là, elle s'est sentie jugée et discriminée à cause de sa religion. Prima, une enseignante noire, s'est aussi sentie ostracisée par les collègues. Ces derniers ont changé, à son insu, la date d'une fête à laquelle elle était conviée et tout le monde était à la fête, sauf elle. Elle dit d'ailleurs que ça a pris beaucoup de temps avant qu'elle soit acceptée comme membre.

Dans les écoles culturellement diversifiées, la situation semble différente. Plus de la moitié des ElFÉ interviewés affirment qu'il y est plus facile de tisser de bonnes relations avec les collègues : "Quand tu te trouves là où tu as la chance d'avoir des immigrants, ce sont eux qui t'intègrent plus facilement que les Québécois »(Olivia). Ces enseignants observent que même les enseignants québécois travaillant dans des écoles culturellement diversifiées sont plus ouverts et accueillants : "Dans la salle des profs, il y avait deux Algériens, un Marocain, un Haïtien et huit Québécois. II y avait une entente extraordinaire, car les enseignants, dans cette école, ont une grande ouverture d'esprit » (Latifa). À l'opposé, dans les écoles culturellement homogènes, cinq enseignants (Latifa, Eva, Olivia, Prima et Michaël) affirment avoir souffert d'indifférence et d'isolement social : "La majorité des enseignants sont québécois et ils viennent à se séparer un peu du reste, de nous. [...] ils s'en foutaient de moi » (Eva).

Enfin, selon certains participants, des qualités personnelles comme l'ouverture d'esprit, la proactivité et le sens de l'humour faciliteraient le contact et l'établissement des liens avec leurs collègues québécois. Ces participants observent que leurs collègues québécois sont plutôt réservés face aux étrangers et estiment que c'est à l'ElFÉ d'initier le contact et de trouver la bonne manière de le faire. C'est aussi grâce à de telles qualités que d'autres enseignants affirment avoir eu de l'aide des collègues. C'est dans ce sens que, Dora, par exemple, dit avoir bénéficié de l'aide d'un collègue québécois expérimenté qui a accepté de l'observer de temps en temps dans sa classe et de lui donner de la rétroaction constructive.

\section{Rapports avec les parents d'élèves}

Cinq participants (Mariya, Rocio, Nadia, Prima et Eva) soulignent que les parents de leurs élèves sont coopératifs. Par exemple, Mariya, enseignante en classe d'accueil auprès d'élèves immigrants nouvellement arrivés au Québec, trouve facile de communiquer avec les parents de ses élèves : « lls sont d'ailleurs très contents de savoir que je suis immigrante comme eux, que je peux donc avoir de l'empathie pour leurs enfants ". Comme on l'a vu précédemment, il est plus facile de créer des liens avec des enseignants et parents immigrants comme eux. Dans le cas de Rocio, enseignante d'espagnol, elle a vécu une relation positive avec les parents, ces derniers lui ayant témoigné leur reconnaissance pour la débrouillardise de leurs enfants en espagnol lors des vacances familiales en Amérique latine.

Par contre, quatre enseignants (Dora, Karim, André et Michaël) ont vécu des expériences plus ou moins négatives avec certains parents d'élèves. Ils racontent que certains parents doutaient de leurs compétences, contestaient les résultats de leurs enfants, refusaient de coopérer ou allaient même se plaindre à la direction plutôt que de communiquer avec eux directement. À cet égard, Dora mentionne : « c'était vraiment comme un choc. Il fallait que je les rassure que je suis nouvelle à l'école, mais pas dans l'enseignement ". Dans le même sens, André affirme: " Des fois, ils [les parents] allaient dire que je n'explique pas assez, que je reste indifférent vis-à-vis des élèves. [...] Ils voulaient que leurs enfants aient de bonnes notes alors qu'ils ne travaillaient pas assez ». Une expérience semblable a été vécue par Karim. Quant à Michaël, il a été choqué d'entendre qu'un parent refusait de le rencontrer parce qu'il est Noir. Ces enseignants estiment d'ailleurs que l'attitude négative de certains parents rend pénible leur intégration professionnelle.

\section{Discussion conclusive}

Cet article avait pour but de décrire et comprendre comment les ElFÉ vivent les relations interpersonnelles et professionnelles lors de leurs premières années d'enseignement en milieu scolaire québécois. Les résultats mettent en évidence que les EIFÉ vivent des expériences négatives, voire douloureuses, mais aussi des expériences positives fondées sur une relation de bienveillance, de respect, d'entraide, de collaboration et d'appréciation. De façon générale, l'expérience de socialisation des 13 enseignants rencontrés varie d'une école à l'autre ou d'une 
commission scolaire à l'autre en fonction du climat de l'école, de l'ouverture de ses membres à la diversité ethnoculturelle et des qualités personnelles de l'EIFÉ.

Selon Fabre et Roussel (2013), les relations entre un nouvel employé et ses collègues et celles avec son directeur n'ont pas les mêmes effets sur sa socialisation. D'après ces auteurs, les interactions positives avec son directeur sont associées « à l'intégration relationnelle, à la maîtrise du fonctionnement politique interne et à l'adhésion aux valeurs et buts organisationnels » (p. 16). Nos résultats vont dans ce sens. Les directions d'école semblent jouer un rôle crucial dans la manière dont se déroule l'intégration réussie ou difficile des EIFÉ au sein de l'équipe-école. En effet, les EIFÉ qui ont bénéficié de l'accueil, de l'orientation, du soutien informationnel et de la reconnaissance de la part de la direction se sont intégrés à l'équipe-école plus facilement que ceux qui ont été laissés à eux-mêmes ou ont eu un accueil plutôt froid, voire méfiant. Certains déplorent d'ailleurs que leurs difficultés en gestion de classe étaient dues à la méconnaissance du fonctionnement de l'école, des règles de conduite auxquelles doivent se conformer les élèves et des ressources auxquelles recourir en cas de besoin. Or, même pour les enseignants non immigrants, les mesures d'accueil et de soutien aux nouveaux enseignants sont essentielles pour faciliter notamment la socialisation organisationnelle et la persévérance dans la profession (Conseil supérieur de l'éducation [CSÉ], 2014 ; Ingersoll, 2012 ; Kutsyuruba et Walker, 2017).

Une recension d'écrits (Martineau et Vallerand, 2006) indique que les rapports entretenus avec la direction d'école influencent l'intégration professionnelle. Cela transparaît dans nos résultats. Les EIFÉ qui ont eu des rapports positifs avec leurs directions soulignent avoir survécu aux difficultés liées à la gestion de classe et à l'enseignement grâce à leur soutien, leur appréciation, leurs rétroactions positives, leurs encouragement et leur évaluation juste. Ainsi, les directions qui accueillent et soutiennent les EIFÉ pour le développement des connaissances sociales et compétences adaptées au nouveau contexte favorisent ce que Morrissette et Demazière (2018) appellent une socialisation " par adhésion et compréhension ». Au contraire, ceux dont les relations sont perçues négativement affirment que leurs directions se montrent davantage menaçantes que bienveillantes, constructives ou aidantes dans les moments difficiles ou conflictuels, ce qui a un impact négatif tant sur leur développement personnel que sur leur intégration professionnelle. Sur le plan professionnel, ces enseignants subissent " une socialisation par imposition et obligation " (Morrissette et Demazière, 2018, p.102). Comme nous l'avons vu, une ElFÉ s'est par exemple sentie jugée et rejetée par la direction à cause de son accent. Étant dans leurs premières années au Québec, les EIFÉ sont encore très sensibles à de telles remarques et se sentent littéralement attaqués dans leur identité et dans leur estime. Soulignons que pour des EIFÉ, les difficultés avec la direction sont vécues de manière prononcée compte tenu de l'écart, en termes de rapport à l'autorité, entre leur culture d'origine et celle du milieu d'accueil où les relations sont moins hiérarchiques. Notre recherche apporte donc un élément nouveau en mettant en évidence le rôle primordial des directions, en tant que supérieur hiérarchique, dans l'intégration et la rétention des EIFÉ.

En outre, les résultats suggèrent que le leadership de la direction, les relations conviviales et collégiales avec les collègues, l'ouverture des membres de la communauté scolaire à la diversité ethnoculturelle et les qualités personnelles de l'EIFÉ constituent des facteurs favorables à une expérience positive d'intégration au sein de l'équipe-école et de socialisation à la culture du milieu. Fabre et Roussel $(2013$, p. 18) soutiennent aussi qu'« un climat convivial au sein d'un groupe de travail incite probablement plus les membres de ce groupe à être ouverts aux nouveaux venus que lorsque les relations entre les membres sont déjà froides et distantes ". Dans le cas des immigrants, plusieurs auteurs (Berry, 1997 ; Buzzanga, 1974 ; Lacroix, 2014) soulignent qu'il leur est impossible de réussir leur intégration sans l'ouverture et la collaboration des membres de la société d'accueil. Nos résultats en témoignent. Les EIFÉ affirment que leur intégration s'est faite plus facilement dans les écoles culturellement diversifiées en raison de l'ouverture de leurs membres à la diversité. Ces résultats confirment d'ailleurs ceux de l'étude de Deters (2006) en Ontario. Par contre, on remarque que les EIFÉ s'intègrent difficilement dans les écoles culturellement moins diversifiées. Tout comme l'indique Lacroix (2014, p. 26), l'entrée d'un EIFÉ dans le milieu scolaire "peut laisser entendre le message de la différence, installer le sentiment de "malaise" ou donner l'impression qu'il y a une "menace". " Césari-Lusso (2008) abonde dans le même sens et note que les individus peuvent résister à l'intégration des étrangers par crainte de perdre leur identité. Certains ElFÉ affirment avoir fait face à l'indifférence, l'isolement social, le racisme et la discrimination. Ces situations sont aussi rapportées dans les études faites en Ontario auprès d'enseignants issus de l'immigration (Duchesne, 2017; Jabouin, 2018; Phillion, 
2003). Il appert qu'une intégration déficiente des EIFÉ au sein de l'équipe-école affecte du même coup leur bienêtre et leur pratique professionnelle. Certains EIFÉ affirment avoir eu peur de demander de l'aide à une direction ou à des collègues qui, dès le départ, leur avaient manifesté une attitude de rejet.

Brillet, Garcia et Montargot (2016) soulignent « l'importance des comportements proactifs des nouveaux arrivants sur le processus de socialisation qui s'opère lors de l'intégration " (p. 109). Les propos de nos répondants vont dans ce sens. Les EIFÉ qui ont pris l'initiative de la communication ou qui ont demandé de l'aide à des collègues ont non seulement développé des relations positives, mais ont de plus bénéficié de soutien pédagogique. Il est ainsi clair, comme le soutient Lacroix (2014), que l'intégration d'un immigrant dépend non seulement de l'ouverture des membres de la communauté d'accueil, mais aussi de sa propre ouverture, de ses efforts et de son souhait d'intégrer sa nouvelle communauté.

Nous venons de voir comment les EIFÉ vivent les relations interpersonnelles et professionnelles lors de leur entrée dans le milieu scolaire québécois et comment celles-ci influencent le déroulement de leur processus de socialisation. Les résultats permettent d'envisager certaines pistes pour mettre en place des mesures susceptibles de faciliter l'accueil et l'intégration socioprofessionnelle des ElFÉ. Par exemple, les directions d'écoles pourraient s'impliquer dans l'intégration de ces enseignants non seulement en leur assurant un accueil personnalisé et un soutien informationnel, mais aussi en instaurant une culture professionnelle de collégialité et d'entraide au sein de l'équipe pédagogique. Par ailleurs, étant donné que "l'acquisition de compétences interculturelles facilite les relations et les échanges entre personnes d'origines et de cultures diverses » (UNESCO, 2013, p. 7), il serait aussi bénéfique d'offrir une formation interculturelle, tant aux enseignants immigrants qu'aux milieux scolaires qui les accueillent. Plus concrètement, les différents acteurs doivent apprendre à s'ouvrir à d'autres cultures, à interagir avec des gens de cultures différentes et à poser un regard critique sur leur propre culture.

\section{Références bibliographiques}

Bachelor, A. et Joshi, P. (1986). La méthode phénoménologique de recherche en psychologie. Québec : Presses de l'Université Laval.

Bascia, N. (1996). Inside and outside: Minority Immigrant Teachers in Canadian schools. Qualitative Studies in Education, 9(2), 151-165.

Bengle, N. M. (1993). L'insertion professionnelle des jeunes : une étude des processus sociopsychologiques dans le secteur des services. Québec : Presses de l'Université Laval.

Berry, J. W. (1997). Immigration, acculturation and adaptation. Applied Psychology: An International Review, 46(1), $5-68$.

Brillet, F., Garcia, J.-F. et Montargot, N. (2016). Conditions de réussite d'une politique d'intégration et développement des compétences : cas d'une entreprise publique en mutation. Revue management et avenir, 88, 105-130.

Buzzanga, M. (1974). L'intégration socioculturelle et ses problèmes. Sherbrooke : Éditions Paulines.

Campoy, E., Waxin, M., Davoine, E., Charles-Pauvers, B., Commeiras, N. et Goudarzi, K. (2005). La socialisation organisationnelle en contexte. In N. Delobbe, O. Herrbach, D. Lacaze, K. Mignonac, Comportement organisationnel (vol. 1) : Contrat psychologique, émotions au travail, socialisation organisationnelle (p. 341393). Bruxelles : De Boeck et Larcier S.A.

Césari-Lusso, V. (2008). Approches interculturelles, approches intraculturelles : Quelles synergies ? Quels défis et quelles ressources pour les acteurs de la formation ? Dans C. Perregaux, P. Dasen, Y. Leanza et A. Gorga (dir.), L'interculturation des savoirs : entre pratiques et théories (p. 97-118). Paris : L'Harmattan.

Collin, S. et Camaraire, L. (2013). Les futurs enseignants issus de l'immigration récente : un modèle de diversité culturelle ? Québec français, 168, 50-51. https://www.erudit.org/fr/revues/qf/2013-n168-

qf0476/68662ac.pdf

Alterstice - Revue Internationale de la Recherche Interculturelle, vol. $8, n^{\circ} 2$ 
Conseil supérieur de l'éducation (CSÉ). (2014). Le développement professionnel, un enrichissement pour toute la profession enseignante. Québec : Gouvernement du Québec.

https://www.cse.gouv.qc.ca/fichiers/documents/publications/Avis/50-0483.pdf

Deters, P. (2006). Immigrant teachers in Canada: Learning the language and culture of a new professional community. Actes du $5^{e}$ Colloque de l'AELFE, 14 au 16 septembre 2006. Saragosse, Espagne : AELFE. http://citeseerx.ist.psu.edu/viewdoc/download?doi=10.1.1.464.4686\&rep=rep1\&type=pdf

Duchesne, C. (2017). Quelles stratégies d'acculturation de nouveaux enseignants issus de l'immigration privilégientils face aux défis culturels et identitaires de leur insertion professionnelle ? Revue canadienne de l'éducation, $40(1), 1-24$.

Duchesne, Ch. (2002). Pratique de socialisation et rétention du personnel : Une étude des professionnels des technologies de l'information et des communications. (Mémoire de maîtrise, Université de Montréal, Montréal). http://biblos.hec.ca/biblio/memoires/m2002no54.pdf

Fabre, C. et Roussel, P. (2013). L'influence des relations interpersonnelles sur la socialisation organisationnelle des jeunes diplômés. Revue de gestion des ressources humaines, 1(87), 2-22.

Giorgi, A. (1997). De la méthode phénoménologique utilisée comme mode de recherche qualitative en sciences humaines : Théorie, pratique et évaluation. Dans J. Poupart, L.Groulx, J.-P. Deslauriers, et A. Laperrière (dir.), La recherche qualitative : enjeux épistémologiques et méthodologiques (p.341-364). Montréal : Gaëtan Morin éditeur.

Gouvernement du Québec. (2001). La formation à l'enseignement : les orientations et les compétences professionnelles. Québec : Ministère de l’Éducation du Québec.

Gouvernement du Québec (2006). Programme de formation de l'école québécoise: enseignement secondaire, premier cycle. Québec: ministère de l'Éducation.

http://www.education.gouv.qc.ca/fileadmin/site_web/documents/education/jeunes/pfeq/PFEQ_presentati on-premier-cycle-secondaire.pdf

Gouvernement du Québec. (2014). Cadre de référence. Accueil et intégration des élèves issus de l'immigration au Québec. Québec : Ministère de l'Éducation, du Loisir et du Sport.

http://www.education.gouv.qc.ca/fileadmin/site_web/documents/education/diversite/Accueillntegration_ 1_PortraitEleves.pdf

Ingersoll, R.-M. (2012). Beginning teacher induction. What the data tell us. Phi delta kappan, 93(8), 47-51.

Jabouin, S. (2018). Trajectoires d'insertion professionnelle des nouveaux enseignants originaires des Caraïbes et d'Afrique subsaharienne (NEOCAS) dans les écoles francophones de l'est de l'Ontario (Thèse de doctorat, Ottawa, Université d’Ottawa). https://ruor.uottawa.ca/handle/10393/38176

Karsenti, T. et Savoie-Zajc, L. (2004). La recherche en éducation : étapes et approches. Sherbrooke : Éditions du CRP.

Kutsyuruba, B. et Walker, K.-D. (2017). The bliss and blisters of early career teaching: a pan-Canadian perspective. Burlington, Ontario : Word and Deed Publishing.

Lacroix, K. (2014). Les relations de travail entre travailleurs natifs et travailleurs immigrants en AbitibiTémiscamingue : recherche-action sur les pratiques des ressources humaines du domaine minier (Mémoire de maîtrise inédit). Université du Québec à Montréal, Montréal.

Lapointe, É. (2013). Examen des contributions d'une approche relationnelle de la socialisation organisationnelle (Thèse de doctorat, Université de Montréal, Montréal). https://papyrus.bib.umontreal.ca/xmlui/handle/1866/10352

Mukamurera, J. (2018). Les préoccupations, le sentiment de compétence et les besoins de soutien professionnels des enseignants débutants : un état de la situation au Québec. Dans J. Mukamurera, J.-F. Desbiens et T. Perez-Roux (dir.), Se développer comme professionnel dans les occupations adressées à autrui : Conditions, modalités et perspectives (p. 189-238). Montréal : Les éditions JFD. 
Martineau, S. et Vallerand, A.-M. (2006). Que peuvent faire les directions d'école pour favoriser l'insertion professionnelle des nouveaux enseignants ? Recherche et Formation, 43, 43-48.

Morrissette, J., Charara, Y. et Boily, A. (2015). L'intégration socioprofessionnelle des enseignants formés à l'étranger : une question d'interaction avec les milieux scolaires. Vivre le primaire, 28(3), 30-31.

Morrissette, J. et Demazière, J. (2018). Dualité des processus de socialisation professionnelle des enseignants formés hors Québec. Entre imposition et appropriation. Alterstice, 8(1), 95-106.

https://www.journal.psy.ulaval.ca/ojs/index.php/ARIRI/article/view/orrissette_Alterstice8\%281\%29

Niyubahwe, A., Mukamurera, J. et Jutras, F. (2018). L'expérience de faire la classe au Québec pour un enseignant immigrant formé à l'étranger. Dans F. Kanouté et J. Charrette (dir.), La diversité ethnoculturelle dans le contexte scolaire québécois : pratiquer le vivre ensemble (p. 177-194). Montréal : Presses de l'Université de Montréal.

Niyubahwe, A., Mukamurera, J. et Jutras, F. (2013). Professional Integration of Immigrant teachers in the school system: A literature review. Revue des sciences de l'éducation de McGill, 48(2), 279-296.

Peeler, E. et Jane, B. (2005). Mentoring: immigrant teachers bridging professional practices. Teaching Education, 16(4), 325-336.

Phillion, J. (2003). Obstacles to accessing the teaching profession for immigrant women. Multicultural Education, 11(1), 41-46.

Portelance, L., Martineau, S. et Presseau, A. (2008). Les représentations des finissants en formation à l'enseignement quant à la collaboration avec les acteurs du milieu scolaire au moment de l'entrée dans la profession. Dans L. Portelance, J. Mukamurera, S. Martineau et C. Gervais (dir.), L'insertion dans le milieu scolaire : une phase cruciale du développement professionnel de l'enseignant (p. 125-144). Québec: Presses de l'Université Laval.

Reid, C. (2005). Global teachers with globite cases. Australian Journal of Education, 49(3), 251-263.

Remennick, L. (2002). Survival of the fittest: Russian immigrant teachers speak about their professional adjustment in Israel. International Migration, 40(1), 99-121.

UNESCO (2013). Compétences interculturelles : cadre conceptuel et opérationnel. https://unesdoc.unesco.org/ark :/48223/pf0000219768_fre

Wang, T. (2003). Cultural dissonance and adaptation: A study of Chinese immigrant teachers coping with cultural differences in Toronto schools (Thèse de doctorat inédite). University of Toronto, Toronto. 